\title{
Low Noise ATRA - Phased Array Measurements of Jet Noise in Flight
}

\author{
Henri Siller* and Timo Schumacher ${ }^{\dagger}$ and Wolfram Hage ${ }^{\ddagger}$ \\ German Aerospace Center (DLR), Berlin, Germany.
}

\begin{abstract}
DLR performed flight tests with retro-fit modifications for noise reduction on its Airbus A320 research aircraft ATRA. Phased array measurements were performed in 2016 with the baseline aircraft and in 2019 with the noise modified aircraft. The array data were processed with acoustic source localisation methods based on the deconvoluton of beamforming maps for moving sources. The resulting sound source maps show the distribution of the sound sources on the aircraft for three emission angles, in the forward arc, overhead, and in the rear arc. Frequency spectra for individual source regions like the engine inlet, the nozzle, and the jet are averaged over all fly-overs in the same configuration for the baseline and the modified aircraft. The comparison of the baseline with the modified aircraft data for the engine nozzle and jet regions confirms that the modified nozzle reduces jet noise.
\end{abstract}

\section{Introduction}

In the DLR internal research project Low Noise ATRA (LNATRA), DLR performed acoustic fly-over measurements with its research aircraft ATRA. The Airbus A320-232 with V2500 engines flew over a large number of single microphones that were distributed over a wide area on both sides of the runway and over a large phased array set up on the end of the runway during several campaigns at the Cochstedt airfield near Magdeburg, Germany. The purpose of these experiments was to test different modifications for noise reduction on the aircraft in flight. Single microphone and phased array measurements were performed for a large number of fly-overs in different configurations with and without modifications for noise reduction applied to the landing gear, the high-lift devices, and the engine nozzles. A companion paper by PottPollenske et al ${ }^{1}$ presents a more general analysis of the effects of the modifications for noise reduction based on the far-field microphone data. This paper presents results of the phased array measurements with a focus on the analysis of the jet-noise measured in flight and on the effect of a profiled trailing edge mounted on the engine nozzles after the base line flight test.

\section{Experimental set-up and procedure}

The aircraft was flown in its baseline configuration in May 2016 and fly-over tests with the aircraft modified for noise reduction were performed in September 2018 and July 2019. First results of the 2016 fly-over test have already been reported by Siller et al..$^{2}$

The microphone array on the ground consisted of 238 microphones that were distributed over an area of 35 by $40 \mathrm{~m}$ and were arranged in a logarithmic spiral that was stretched in the direction of flight. The microphone data were synchronized with the flight log of the aircraft using a GPS time signal. An array of laser distance meters on the ground was used to determine the basic aircraft trajectory parameters like the ground speed, the vertical speed and the altitude as well as trigger times when the aircraft passed over the array. The experimental procedure was similar to that described in Siller et al. ${ }^{3}$

\footnotetext{
*Senior Scientist, German Aerospace Center (DLR), Institute of Propulsion Technology, Department of Engine Acoustics, Berlin, Germany

$\dagger$ Research Scientist, Technische Universität, Berlin, Germany

${ }^{\ddagger}$ Research Scientist, German Aerospace Center (DLR), Institute of Propulsion Technology, Department of Engine Acoustics, Berlin, Germany
} 


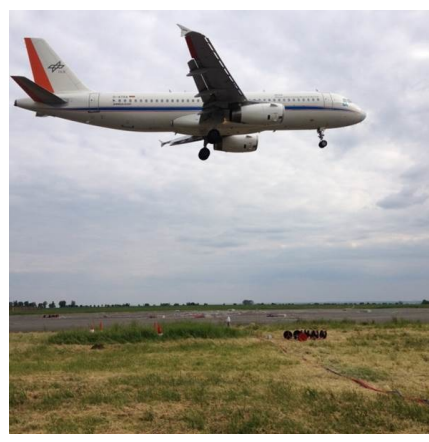

(a)

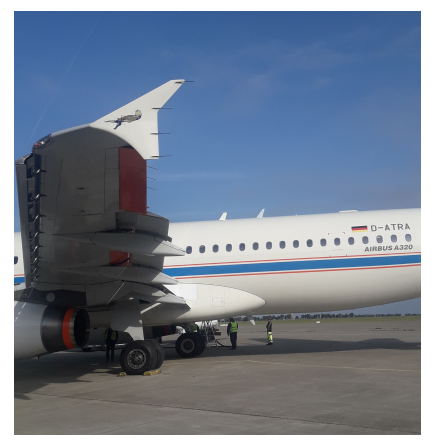

(b)

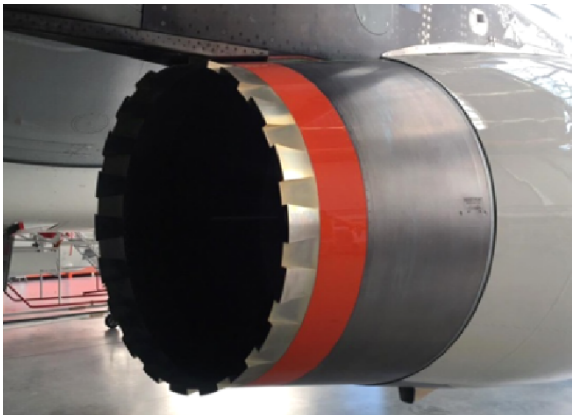

(c)

Figure 1: a) The DLR ATRA in its base line configuration flying over the phased array at Cochstedt airfield in May 2016, b) the ATRA in 2019 with the modified engine nozzles and high-lift devices, and c) the noise modified engine nozzle with the profiled trailing edge mounted on the ATRA for the 2019 fly-over tests.

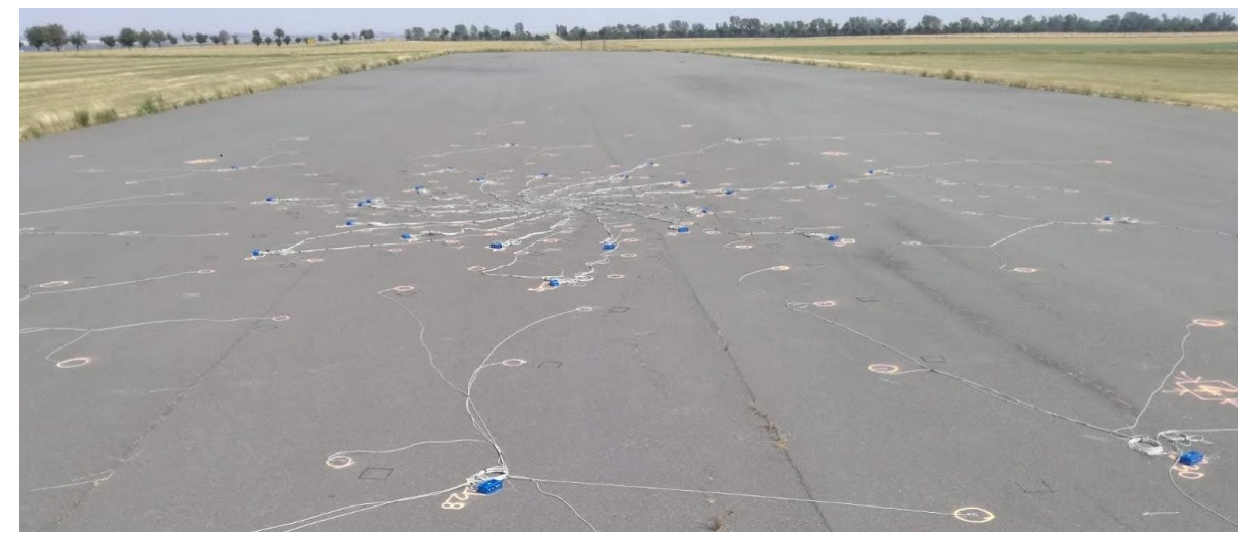

Figure 2: Set-up of the phased array on the runway for the 2019 fly-over tests.

\section{Data processing}

In a first pass of the analysis, the microphone array data were processed using the classical beamforming algorithm for moving sources ${ }^{4}$ The resulting source maps were used to iteratively adjust the trajectory in order to properly center the aircraft on the interrogation grid that moves with the aircraft. Such corrections are necessary in order to compensate uncertainties in the trajectory data and deviations from a straight propagation path between the microphones and the aircraft due to the effects of wind or thermal stratification. After the corrections of the aircraft position, the proper source localization was performed with the hybrid deconvolution algorithm that is implemented in the DLR code ProSigMA ${ }^{5}$ From the results of this analysis, source maps were evaluated in one-third-octave bands at three emission angles in the forward arc, overhead, and in the rear arc.

\section{Results}

Figure 3 presents examples of sound source maps for a fly-over in the landing configuration with an airspeed of about $170 \mathrm{kts}$, engines running in flight idle, fully deployed slats and flaps $\left(27^{\circ}\right.$ and $\left.40^{\circ}\right)$, and the landing gear extended. The maps shown here, for the $1 \mathrm{kHz}$ and the $3.15 \mathrm{kHz}$ one-third-octave bands, were calculated for the time interval when the aircraft was directly above the array. The most prominent sources are the nose and the main landing gear, but also the distributed sources along the high-lift system on the wings.

For a proper comparison of the microphone array deconvolution maps obtained from different fly-overs, a scaling of the flight altitude to a reference altitude, and a compensation of the atmospheric absorption of 

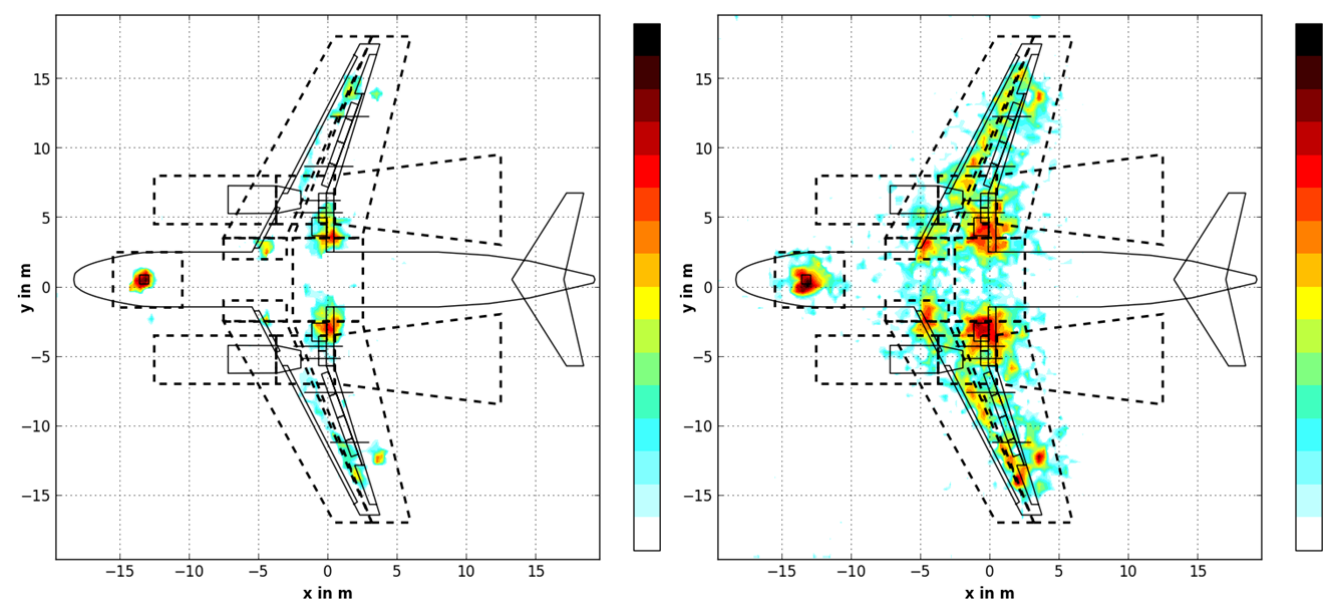

Figure 3: Sound source maps for a fly-over in the simulated landing configuration with the aircraft directly above the array in the $1 \mathrm{kHz}$ (left) and the $3.15 \mathrm{kHz}$ (right) one-third-octave bands, the dynamic range of the maps is $16 \mathrm{~dB}$.

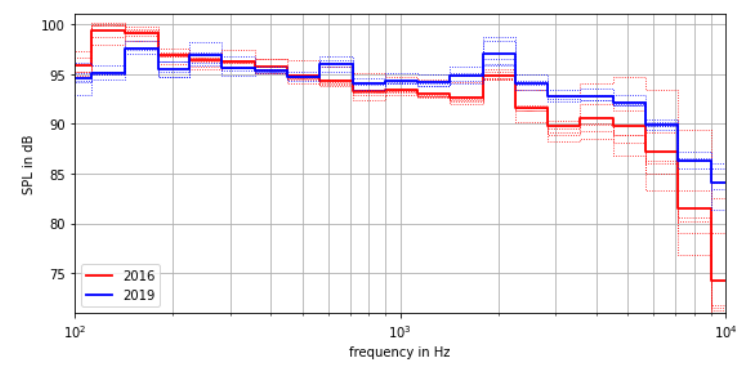

(a) Farfield TOB Spectrum, $\theta=60^{\circ}$

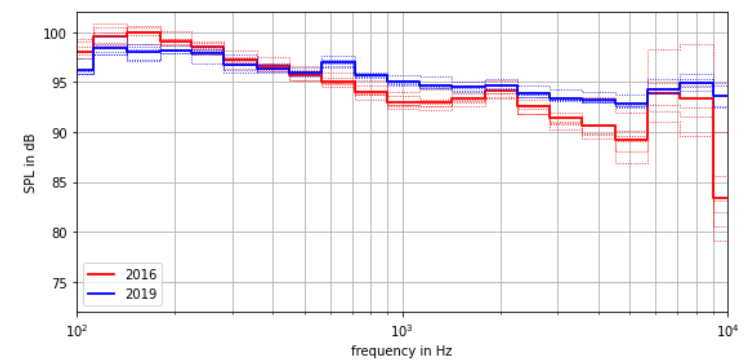

(c) Farfield TOB Spectrum, $\theta=90^{\circ}$

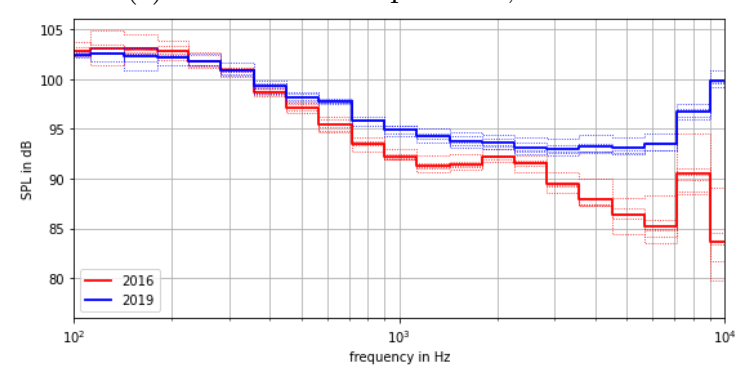

(e) Farfield TOB Spectrum, $\theta=120^{\circ}$

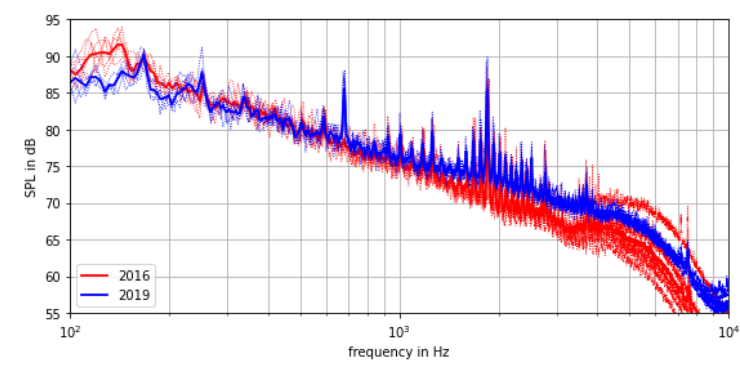

(b) Farfield Narrow Band Spectrum, $\theta=60^{\circ}$

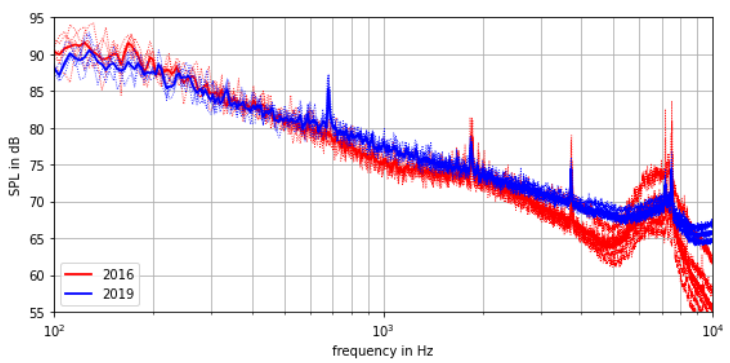

(d) Farfield Narrow Band Spectrum, $\theta=90^{\circ}$

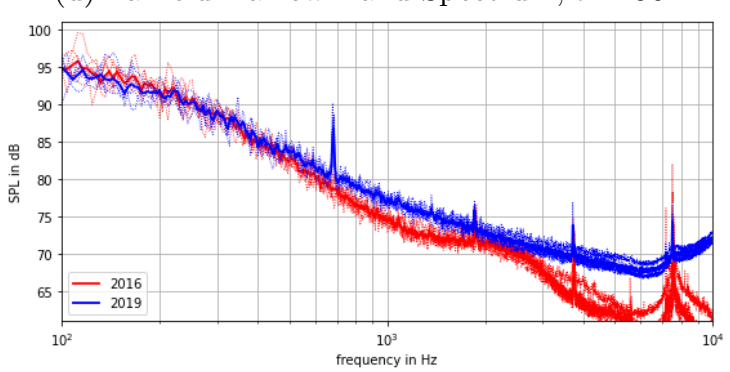

(f) Farfield Narrow Band Spectrum, $\theta=120^{\circ}$

Figure 4: Dedopplerized Spectra from the baseline and the modified aircraft for a take-off configuration with engine speeds of $N 1=90 \%$ and flight speeds of $U_{\text {flight }}=73 \mathrm{~m} / \mathrm{s}$. The bold lines: averaged levels, thin, dotted lines: individual flyovers. 


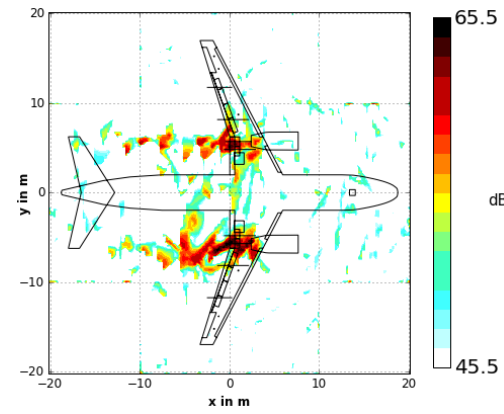

(a) $f=200 \mathrm{~Hz}, \theta=60^{\circ}$

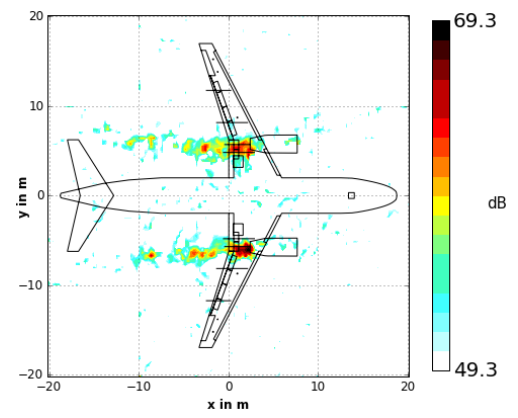

(d) $f=315 \mathrm{~Hz}, \theta=60^{\circ}$

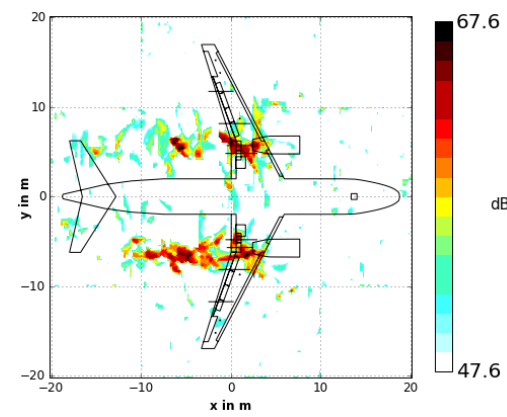

(b) $f=200 \mathrm{~Hz}, \theta=90^{\circ}$

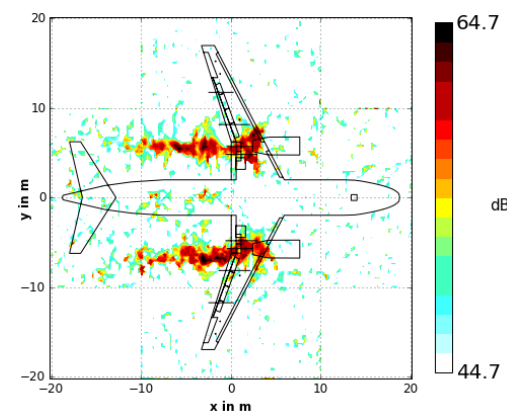

(e) $f=315 \mathrm{~Hz}, \theta=90^{\circ}$

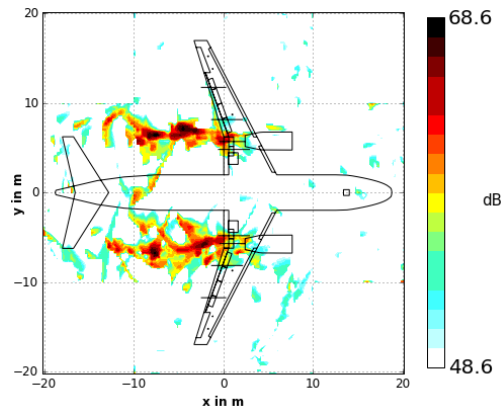

(c) $f=200 \mathrm{~Hz}, \theta=120^{\circ}$

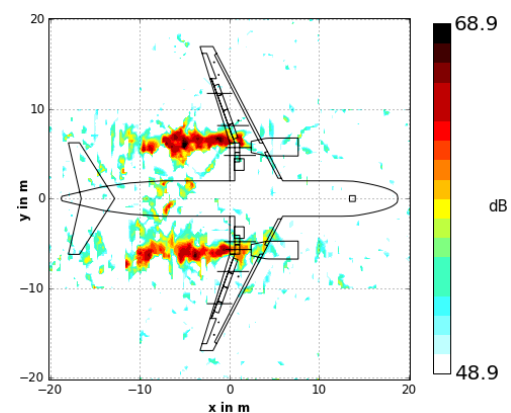

(f) $f=315 \mathrm{~Hz}, \theta=120^{\circ}$

Figure 5: Sound source maps in the $f=200 \mathrm{~Hz}$ and $f=315 \mathrm{~Hz}$ one-third-octave bands from the 2016 baseline test for a take-off with an engine speed of $N 1=90 \%$ and a flight speed of $U_{\text {flight }}=73 \mathrm{~m} / \mathrm{s}$ in the forward $\operatorname{arc}(\mathrm{a}, \mathrm{d})$, overhead $(\mathrm{b}, \mathrm{e})$, and in the rear $\operatorname{arc}(\mathrm{c}, \mathrm{f})$

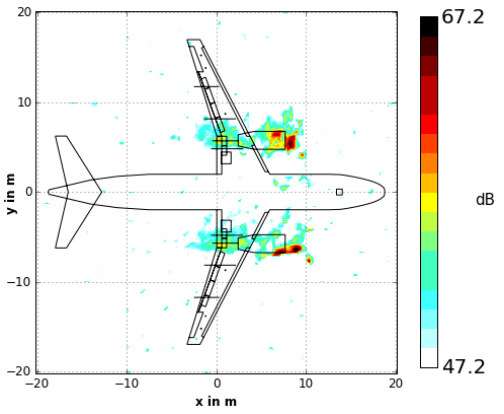

(a) $f=2 \mathrm{kHz}, \theta=60^{\circ}$

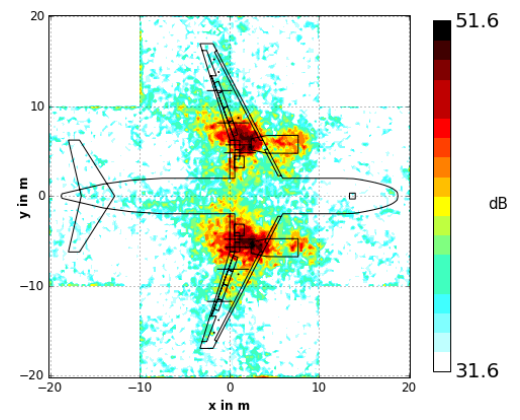

(d) $f=6.3 \mathrm{kHz}, \theta=60^{\circ}$

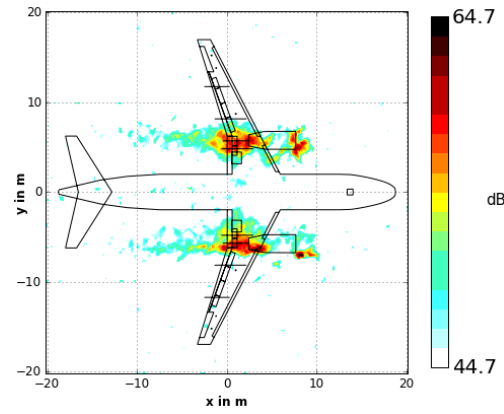

(b) $f=2 \mathrm{kHz}, \theta=90^{\circ}$

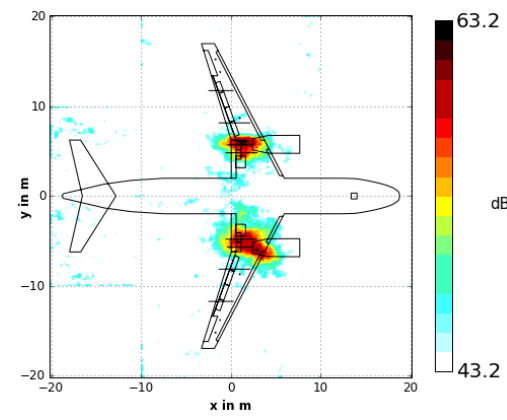

(e) $f=6.3 \mathrm{kHz}, \theta=90^{\circ}$

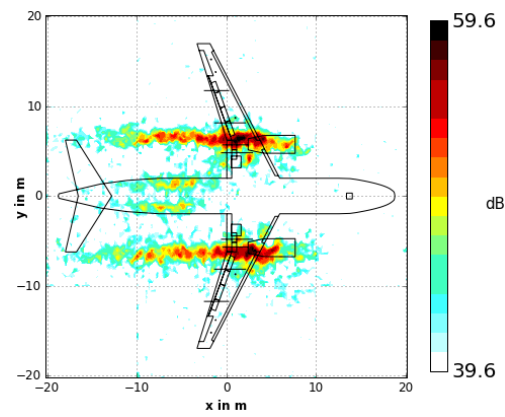

(c) $f=2 \mathrm{kHz}, \theta=120^{\circ}$

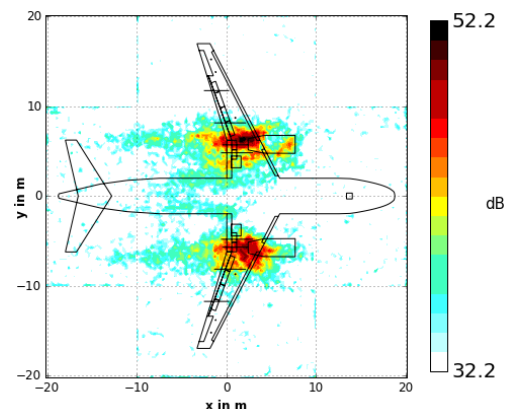

(f) $f=6.3 \mathrm{kHz}, \theta=120^{\circ}$

Figure 6: Sound source maps in one-third-octave bands that contain tonal components of the fan and turbine from the 2016 baseline test for a take-off with an engine speed of $N 1=90 \%$ and a flight speed of $U_{\text {flight }}=73$ $\mathrm{m} / \mathrm{s}$ in the forward $\operatorname{arc}(\mathrm{a}, \mathrm{d})$, overhead $(\mathrm{b}, \mathrm{e})$, and in the rear $\operatorname{arc}(\mathrm{c}, \mathrm{f})$ 


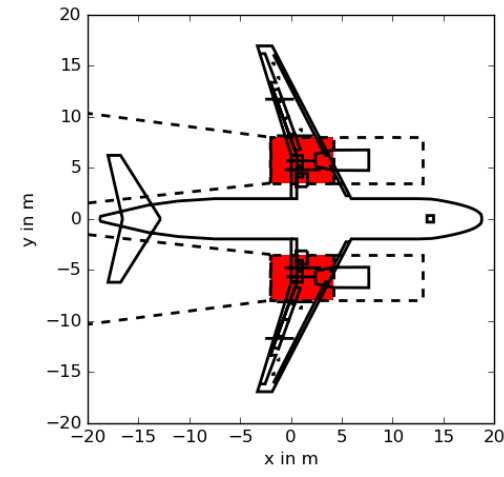

(a) Integration region Nozzles

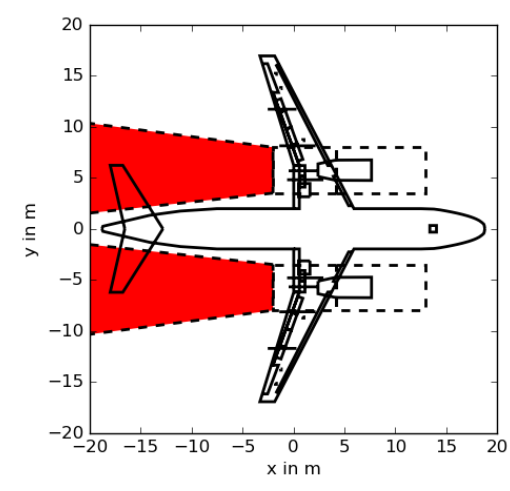

(b) Integration region Jets

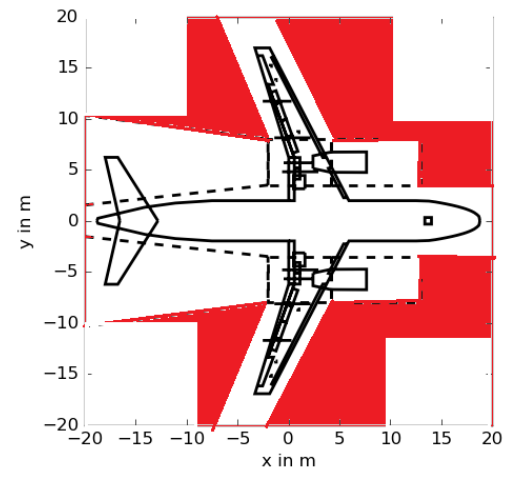

(c) Integration region Rest

Figure 7: Source integration areas for different aircraft components and the area outside the aircraft.

sound have to be performed. Also, the sound powers in the jet need to be scaled with the difference between the flight speed and the jet speed $U_{\text {jet }}-U_{\text {flight }}$ scaled with the speed of sound $a$. The jet velocities are not available from the flight log data and were calculated using the simulation code of the DLR institute of propulsion technology $\sqrt{6}$ based on the the altitude, static and total pressures, and temperature from the flight recorder data at the time when the aircraft passed over the microphone array. For practical purposes, the jet noise contribution can be scaled with the relative jet speed normalized with the speed of sound $\left(U_{\text {jet }}-U_{\text {flight }}\right) / a$ to a power of eight or by using the more elaborate method proposed by Michel ${ }^{[7}$ After applying these normalisations, the deconvolution results can be compared between different fly-overs and averaged over similar fly-overs in the same configuration.

In the following, we concentrate on the analysis of data from fly-overs in a take-off configuration with an engine speed of $N 1=90 \%$ and a flight speed of $U_{\text {flight }}=73 \mathrm{~m} / \mathrm{s}$. In this configuration, six fly-overs were recorded in the 2016 baseline configuration flight test and five fly-overs with the modified aircraft were recorded in 2019.

Figure 4 presents the Doppler compensated far-field spectra which were calculated for each of the array microphones individually and then averaged over all microphones in the array. The spectra are presented for three emisson angles $\theta=60^{\circ}, 90^{\circ}$, and $120^{\circ}$, when the aircraft is approaching, above the array, and flying away, respectively. The emisson angle $\theta$ is the angle between the aircraft velocity vector and a line from the aircraft center to the array center. The data segments used for each emission angles are centered around the nominal angle with an interval size of $\theta \pm 10^{\circ}$. In figure 4 the individual frequency spectra of the fly-overs in the baseline and in the modified configuration are shown as thin dotted lines and the spectra averaged over all fly-overs in the baseline or the modified configuration as bold solid lines. The results show a relative good repeatability of the source levels for fly-overs in the same configuration.

The sound source maps for the take-off in the baseline configuration are presented in figures 5 and 6 . Figure 5 (a-c) shows the maps in the $200 \mathrm{~Hz}$ one-third octave band. Here, the Rayleigh limit which determines the capability of an array to separate sources, is at $9.3 \mathrm{~m}$ source separation at $200 \mathrm{~Hz}$ for the array with a diameter of $40 \mathrm{~m}$. The maps show that the deconvolution method is able to resolve sources in the jet, where the classical beamforming maps would just show one large central source. The resolution improves for higher frequencies (see figure 5 (d-f) with the $315 \mathrm{~Hz}$ maps).

The deconvolution algorithm in the DLR ProSigMA code is based on a model of broadband sources ${ }^{5}$ However, the method is also able to localize tonal sources: figure 6 presents the maps in the $2 \mathrm{kHz}$ band that contains the blade passing frequency of the fan and the maps in the $6.3 \mathrm{kHz}$ band with the turbine tones.

In the post-processing of the results, the interrogation grid for the calculation was divided into source regions, which are indicated in the source maps with dashed lines (see figure 33). For the analysis of the engine noise contribution, source regions were defined that cover the engine inlets, the nozzles (figure 7a), and the free-jet regions downstream of the nozzles (see figure 7b). Further regions included the sources of the fuselage and wings. All sources not associated with any region are shown in figure $7 \mathrm{c}$ and called Rest, whereas the sum of all regions is labeled Total Aircraft. The source powers in these regions can be integrated in order to obtain the total contribution of a specific source region. The resulting source region powers can 

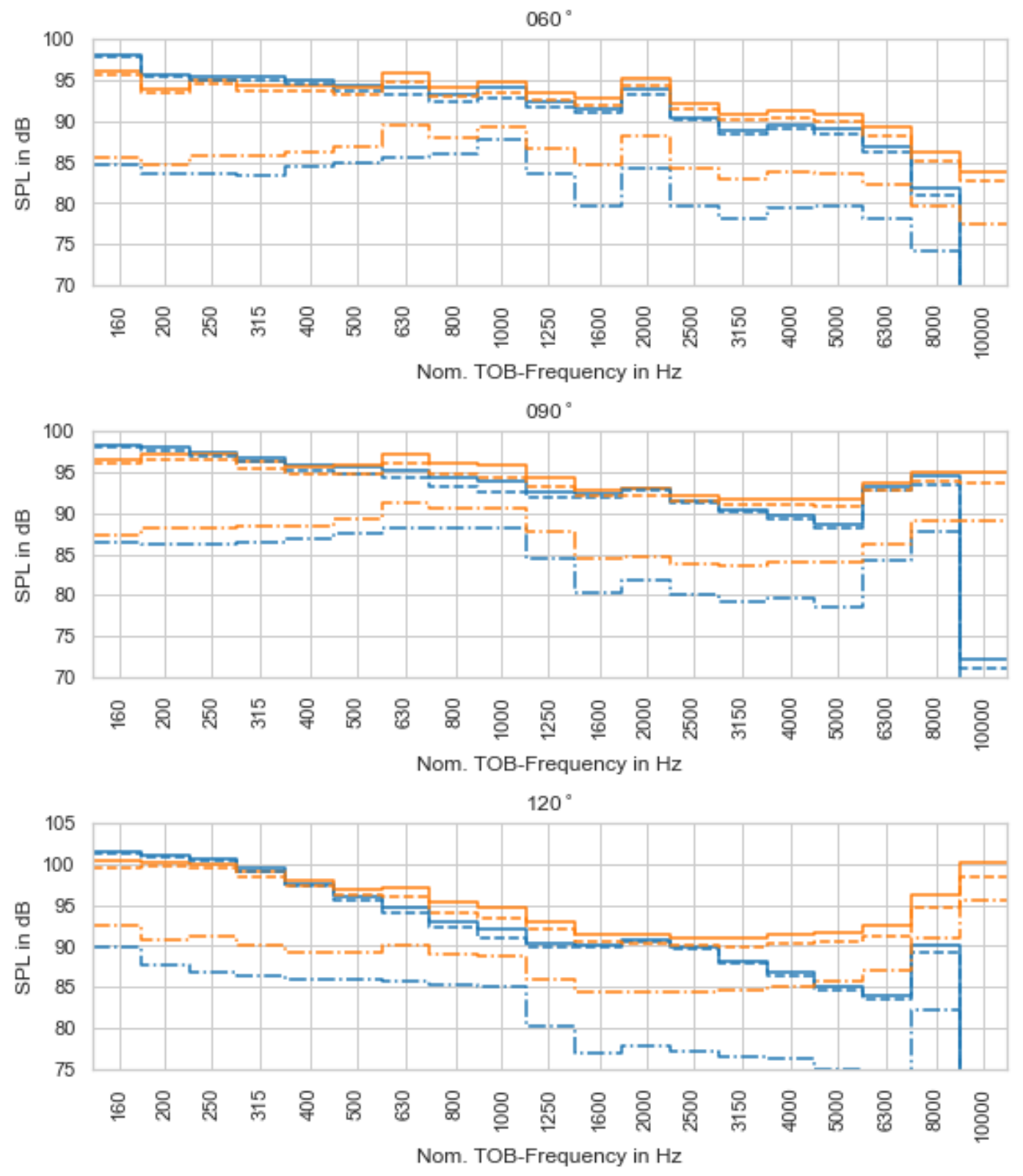

$\begin{array}{lll}\text { - Baseline - TotalMap } & - & \text { Low Noise - TotalMap } \\ \text {--- Baseline - TotalAircraft } & --- & \text { Low Noise - TotalAircraft } \\ \text {-- - Baseline - Rest } & -\cdot-\text { Low Noise - Rest }\end{array}$

Figure 8: Comparison of the source integration spectra of the TotalMap, the TotalAircraft and the Rest regions. 

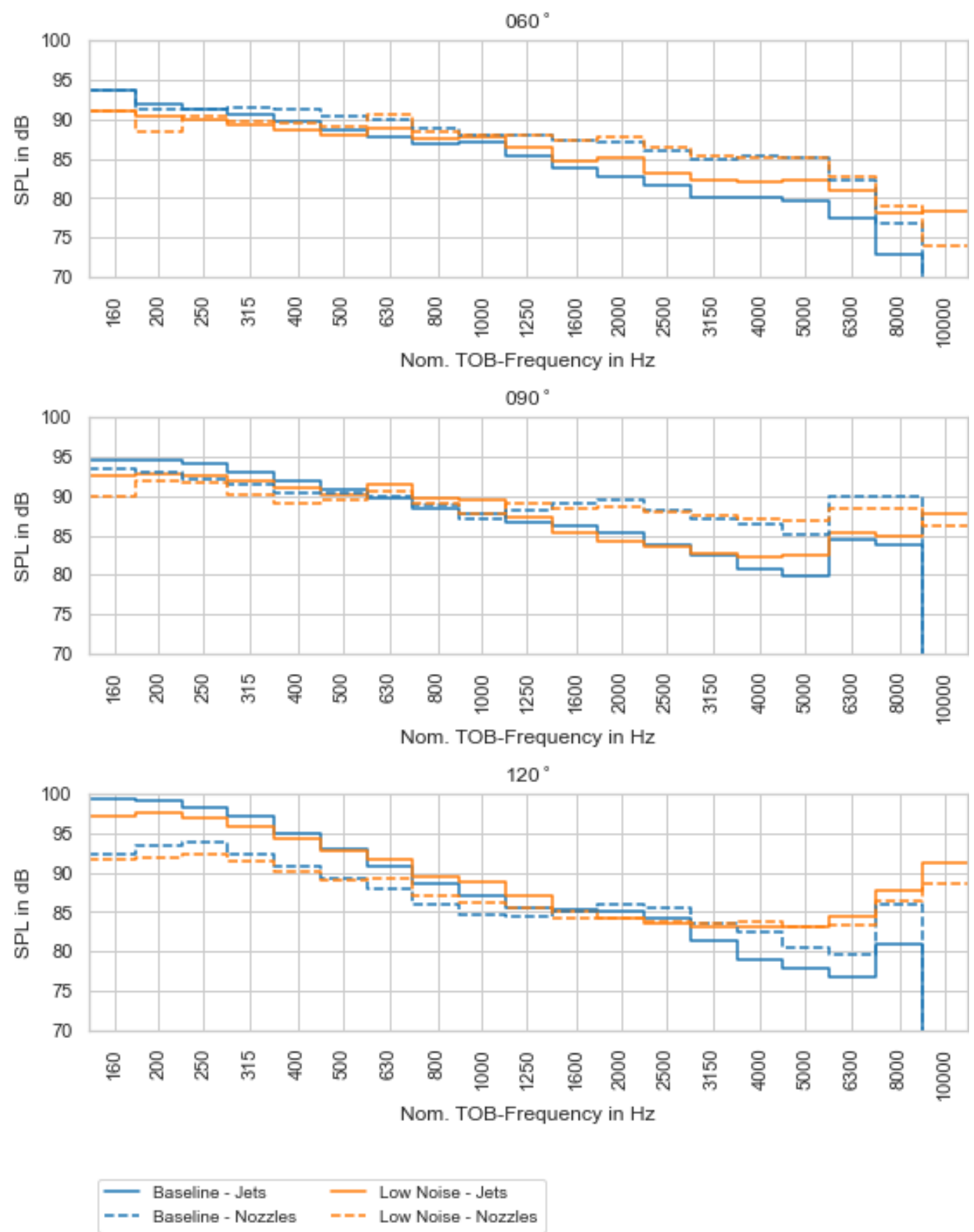

Figure 9: Comparison of the source integration spectra of the Jets and Nozzles regions. 


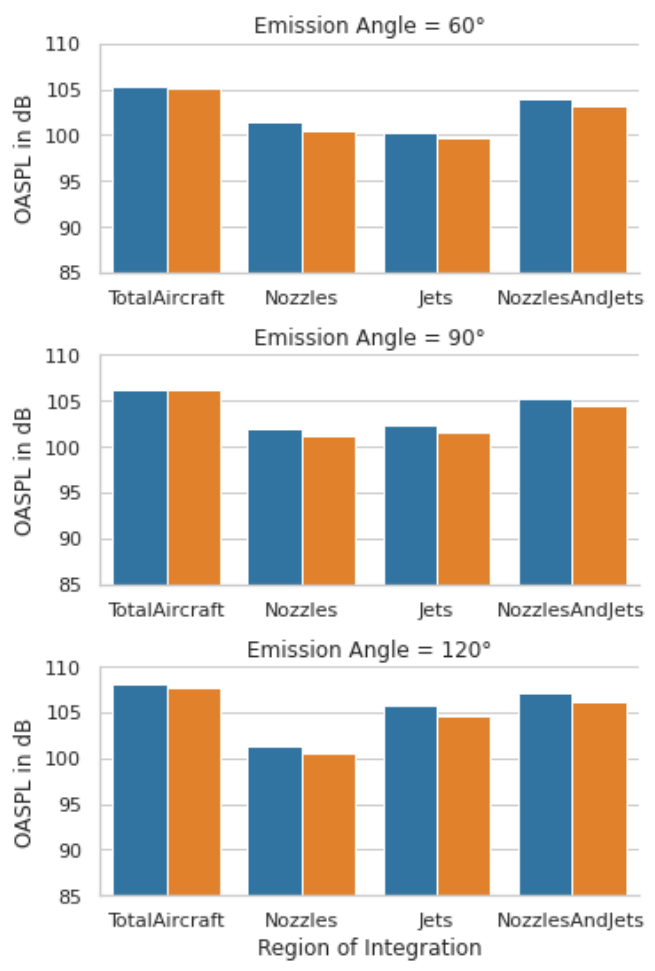

Figure 10: Comparison of the Overall Sound Pressure Levels (OASPL) of the baseline (blue) and the modified aircraft (orange) in the frequency range from $160 \mathrm{~Hz}$ to $4 \mathrm{kHz}$.

be averaged over several fly-overs in the same flight configuration in order to improve the statistics of the analysis. In most configurations flown for the LNATRA flight test, up to six repeated fly-overs are available per configuration.

The resulting spectra where converted from narrow bands to third octave bands and plotted. Figure 8 shows the total acoustic energy of the source map and the components areas Total Aircraft, i.e. the sum over all integration regions on the aircraft and Rest, the area inside the map but outside the aircraft, see figure 7. We can see that most energy is localized within close proximity to the aircraft or its Jets. The dynamic range between the source power in the total map area and the Rest region is between 6 and $10 \mathrm{~dB}$.

The source integration results for the jet and nozzle regions (defined in figures $7 \mathrm{~b}$ and $7 \mathrm{a}$, repectively) are compared in figure 9. The baseline configuration features louder low-frequency jet noise, compared with the modified nozzle configuration. For both the baseline and the low noise modified configurations, the low frequency noise in the Jets regions is strongest in the rear arc, at $\theta=120^{\circ}$. The Nozzles regions show higher source levels than the Jets regions at higher frequencies in the forward arc and overhead (i.e. for $\theta=60^{\circ}$ and $\theta=90^{\circ}$. The cross-over frequency between stronger contributions from the jet or the nozzle is around $1 \mathrm{kHz}$. The nozzle edge modification incurs a high frequency noise penalty in the Jets regions in the forward arc at $\theta=60^{\circ}$. Contrary to expectation, this excess high frequency noise occurs in the jet region downstream of the nozzle and not in the area close to the nozzles.

In order to further concentrate the data, the power in source regions was integrated from $160 \mathrm{~Hz}$ to 4 $\mathrm{kHz}$ (excluding turbine tone frequencies at 6.3 and $8 \mathrm{kHz}$ ) in order to obtain overall sound pressure levels (OASPL). Figure 10 compares the OASPL of the Total Aircraft region with the OASPL in the Jets, the Nozzles, and the combined jet and nozzle areas between the baseline and the noise modified configurations. There is a small noise benefit of the modified nozzle at all emission angles. The difference is greater in the jets and nozzles regions than at full aircraft level. A proper appraisal of the noise benefit of the modified nozzle would require a more detailed analysis over the full range of emission angles between $30^{\circ}<\theta<150^{\circ}$. Pott-Pollenske et al ${ }^{[1]}$ have performed a proper analysis based on far-field microphones and calculated a total noise benefit of the low noise nozzle of about 0.5 EPNdB. 


\section{Conclusions and outlook}

The DLR research aicraft ATRA was flown in two flight tests in the baseline and in a configuration with modifications for noise reduction. For fly-overs in the take-off configuration, the phased array were processed with the DLR beamforming and deconvolution code ProSigMA. Noise source maps were calculated and the source powers were integrated over source regions in the areas covering the engine jets, nozzles, and inlets. This process yields frequency spectra of the sound source levels of the specific source regions. The dynamic range of the integration results is between 6 and $10 \mathrm{~dB}$ between the source power calculated over the whole area of the source map compared to the source regions on the aircraft. The analysis of the integration areas for the engine jets and nozzles provides more detailed information on the effects of the trailing edge modification of the engine nozzle than an analysis based on farfield microphone data. The source integration allows to directly compare the sound powers in different source regions for different configurations. The jet noise in the frequencies in the $400 \mathrm{~Hz}$ one-third-octave band and below is reduced by the nozzle modification. As it can be expected, the sound power in the jet region is stronger than near the engine nozzle in the low frequency range. Above a cross-over frequency between 1000 and $1250 \mathrm{~Hz}$, the source power in the nozzle region is stronger than further downstream in the jets.

A high-frequency noise penalty induced by the nozzle trailing edge is observed in the forward radiation arc in the one-third-octave bands from $1250 \mathrm{~Hz}$ to $5 \mathrm{kHz}$. These results are a first attempt in trying to understand the effect and the noise reduction mechanism of the profiled nozzle. A more detailed analyis over the full range of emission angles from $30^{\circ}$ to $150^{\circ}$ with a more elaborate cut of integration regions, e.g. a nozzle region that is only one or two nozzle diameters long and a separation of the jet area in to slices based on the relative distance to the nozzle could provide more valuable results.

\section{References}

\footnotetext{
${ }^{1}$ Pott-Pollenske, M., "Aircraft Noise Reduction by means off Retro-Fit Technologies," 27rd AIAA/CEAS Aeroacoustics Conference, AIAA AVIATION Forum, 2021, AIAA paper 2021-2117.

${ }^{2}$ Siller, H. A., Bassetti, A., Hage, W., and Funke, S., "Measurements of the noise emitted by the V2500 engines in flight and in static measurements on the ground," 23rd AIAA/CEAS Aeroacoustics Conference, AIAA AVIATION Forum, (AIAA 2017-3844) https://doi.org/10.2514/6.2017-3844, Juni 2017.

${ }^{3}$ Siller, H., "Localisation of sound sources on aircraft in flight," Proceedings on CD of the 4th Berlin Beamforming Conference, 22-23 February 2012, GFaI, Gesellschaft zu Förderung angewandter Informatik e.V., Berlin, February 2012.

${ }^{4}$ Piet, J.-F., Michel, U., and Böhning, P., "Localization of the acoustic sources of the A340 with a large phased microphone array during flight tests," 8th AIAA/CEAS Aeroacoustics Conference, Breckenridge, Co, 17-19 June 2002, 2002.

${ }^{5}$ Guérin, S. and Weckmüller, C., "Frequency-domain reconstruction of the point-spread function for moving sources," Proceedings on CD of the 2nd Berlin Beamforming Conference, 19-20 February, 2008, GFaI, Gesellschaft zu Förderung angewandter Informatik e.V., Berlin, 2008.

${ }^{6}$ Wolters, F., Becker, R.-G., Schnell, R., and Ebel, P.-B., "Engine performance simulation of the integrated V2527-Engine Fan," 54th AIAA Aerospace Sciences Meeting, 2016.

${ }^{7}$ Michel, U., "Prediction of jet mixing noise in flight from static tests," 22nd AIAA/CEAS Aeroacoustics Conference, 2020, AIAA paper 2016-2807.
}

\section{Acknowledgements}

Some of the research leading to the presented results has been supported by the European Community's Seventh Framework Program for research, technological development and demonstration under grant agreement no. 604013 . 